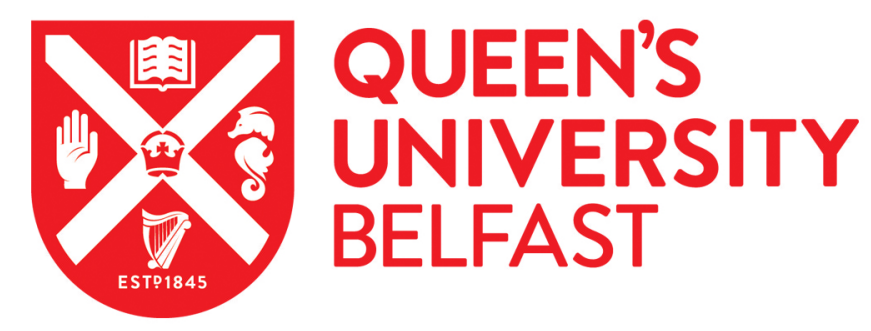

\title{
Susceptibility of streptozotocin-induced diabetic rat retinal function and ocular blood flow to acute intraocular pressure challenge.
}

Wong, V. H. Y., Vingrys, A. J., Jobling, A. I., \& Bui, B. V. (2013). Susceptibility of streptozotocin-induced diabetic rat retinal function and ocular blood flow to acute intraocular pressure challenge. Investigative ophthalmology \& visual science, 54(3), 2133-2141. https://doi.org/10.1167/iovs.13-11595

\section{Published in:}

Investigative ophthalmology \& visual science

\section{Document Version:}

Publisher's PDF, also known as Version of record

Queen's University Belfast - Research Portal:

Link to publication record in Queen's University Belfast Research Portal

\section{Publisher rights}

Copyright 2013 The Association for Research in Vision and Ophthalmology, Inc. This work is made available online in accordance with the publisher's policies. Please refer to any applicable terms of use of the publisher.

\section{General rights}

Copyright for the publications made accessible via the Queen's University Belfast Research Portal is retained by the author(s) and / or other copyright owners and it is a condition of accessing these publications that users recognise and abide by the legal requirements associated with these rights.

Take down policy

The Research Portal is Queen's institutional repository that provides access to Queen's research output. Every effort has been made to ensure that content in the Research Portal does not infringe any person's rights, or applicable UK laws. If you discover content in the Research Portal that you believe breaches copyright or violates any law, please contact openaccess@qub.ac.uk. 


\title{
Susceptibility of Streptozotocin-Induced Diabetic Rat Retinal Function and Ocular Blood Flow to Acute Intraocular Pressure Challenge
}

\author{
Vickie H. Y. Wong, ${ }^{1}$ Algis J. Vingrys, ${ }^{1}$ Andrew I. Jobling, ${ }^{2}$ and Bang V. Bui ${ }^{1}$
}

PurPose. To consider the hypothesis that streptozotocin (STZ)induced hyperglycemia renders rat retinal function and ocular blood flow more susceptible to acute IOP challenge.

Methods. Retinal function (electroretinogram [ERG]) was measured during acute IOP challenge (10-100 $\mathrm{mm} \mathrm{Hg}$, increments of $5 \mathrm{~mm} \mathrm{Hg}, 3$ minutes per step, vitreal cannulation) in adult Long-Evans rats ( 6 weeks old; citrate: $n$ $=6$, STZ: $n=10) 4$ weeks after citrate buffer or STZ $(65 \mathrm{mg} / \mathrm{kg}$, blood glucose $>15 \mathrm{mM}$ ) injection. At each IOP, dim and bright flash $\left(-4.56,-1.72 \log\right.$ cd.s. $\left.{ }^{-2}\right)$ ERG responses were recorded to measure inner retinal and ON-bipolar cell function, respectively. Ocular blood flow (laser Doppler flowmetry; citrate: $n=6$, STZ: $n=10$ ) was also measured during acute IOP challenge. Retinas were isolated for quantitative PCR analysis of nitric oxide synthase mRNA expression (endothelial, eNos; inducible, iNos; neuronal, nNos).

Results. STZ-induced diabetes increased the susceptibility of inner retinal (IOP at 50\% response, 60.1, CI: 57.0-62.0 mm Hg versus citrate: 67.5 , CI: $62.1-72.4 \mathrm{~mm} \mathrm{Hg}$ ) and ON-bipolar cell function (STZ: 60.3 , CI: $58.0-62.8 \mathrm{~mm} \mathrm{Hg}$ versus citrate: 65.1 , CI: 61.9-68.6 mm Hg) and ocular blood flow (43.9, CI: 40.846.8 versus citrate: $53.4, \mathrm{CI}: 50.7-56.1 \mathrm{~mm} \mathrm{Hg}$ ) to IOP challenge. Citrate eyes showed elevated eNos mRNA (+49.7\%) after IOP stress, an effect not found in STZ-diabetic eyes $(-5.7 \%, P<0.03)$. No difference was observed for iNos or nNos $(P>0.05)$ following IOP elevation.

Conclusions. STZ-induced diabetes increased functional susceptibility during acute IOP challenge. This functional vulnerability is associated with a reduced capacity for diabetic eyes to upregulate eNos expression and to autoregulate blood flow in response to stress. (Invest Ophthalmol Vis Sci. 2013; 54:2133-2141) DOI:10.1167/iovs.13-11595

A major complication affecting many patients with diabetes is retinopathy, which is clinically characterized by vascular abnormalities, such as microaneurysms, hemorrhages, and neovascularization, which can lead to vision loss. ${ }^{1}$ There is growing evidence that neuronal abnormalities might precede

From the Departments of ${ }^{1}$ Optometry and Vision Sciences and ${ }^{2}$ Anatomy and Neuroscience, The University of Melbourne, Parkville, Victoria, Australia.

Supported by National Health and Medical Research Council of Australia Project Grant 566570 (BVB, AJV).

Submitted for publication January 2, 2013; revised February 3 and 12, 2013; accepted February 15, 2013.

Disclosure: V.H.Y. Wong, None; A.J. Vingrys, None; A.I. Jobling, None; B.V. Bui, None

Corresponding author: Bang V. Bui, Department of Optometry and Vision Sciences, The University of Melbourne, Parkville, 3010, VIC, Australia; bvb@unimelb.edu.au. microangiopathy. ${ }^{2}$ For example, functional assessment of the retina using electrophysiological measures reveals changes in function ${ }^{3,4}$ prior to clinically detectable microangiopathy. Indeed, Bearse et al. ${ }^{5}$ and Harrison et al. ${ }^{6}$ provide evidence that regions of the retina showing functional deficits are more likely to go on to show signs of microangiopathy. The presence of neuronal dysfunction may reflect subtle changes to the vasculature not readily detectable clinically. Evidence in clinical studies (none or mild nonproliferative diabetic retinopathy) ${ }^{7-9}$ and in animal models suggest that changes in retinal blood flow, ${ }^{10}$ impaired blood vessel reactivity, ${ }^{11-13}$ and autoregulation ${ }^{14}$ occur early in the course of the disease.

In addition to the development of diabetic retinopathy, the presence of vascular dysfunction or some inherent neuronal weakness may place diabetic eyes at greater risk of other neurodegenerative diseases, such as glaucoma. Glaucoma affects an estimated 60 million people worldwide ${ }^{15}$ and is characterized by a progressive loss of retinal ganglion cells. The most well-defined risk factor for glaucoma is elevated intraocular pressure (IOP). Epidemiological studies have returned conflicting outcomes as to whether diabetes increases the risk of glaucoma (as reviewed in Wong et al. $^{16}$ ). However, these studies did not set out to directly consider the association between diabetes and glaucoma. Laboratory studies provide some evidence in support of this possibility. Kanamori et al. $^{17}$ reported that streptozotocin (STZ)-induced hyperglycemic rats showed greater apoptosis of retinal neurons after exposure to chronic IOP elevation compared with normoglycemic rats. Kohzaki et al. ${ }^{18}$ showed a reduction in the recovery of retinal ganglion cell responses 1 week after a single episode of IOP challenge, an effect not evident in citrate control rats. Whether this increased susceptibility arises from impairment to the vascular system or via a deficit in retinal neurons is not clear.

This study considered the hypothesis that STZ-induced hyperglycemia increases susceptibility of the electroretinogram and ocular blood flow to acute IOP challenge. In the same cohort of animals, we also consider a potential mechanism by comparing the effect of IOP challenge on gene expression of nitric oxide synthase (Nos)-isoenzymes (endothelial, eNos; inducible, iNos; neuronal, nNos) in diabetic and control eyes.

\section{Methods}

\section{Animals}

All experimental procedures abide with the ARVO Statement for the Use of Animals in Ophthalmic and Vision Research and the National Health and Medical Research Council's Australian Code of Practice for the care and use of animals for scientific purposes. Animal ethics approval was obtained from the Faculty of Science Animal Ethics Committee at The University of Melbourne. Hyperglycemia was 
TABLE 1. Metabolic Parameters (Blood Glucose, IOP, and Systolic Blood Pressure; Mean \pm SEM) of Control and Diabetic Animals

\begin{tabular}{|c|c|c|c|c|c|c|c|}
\hline \multirow[b]{2}{*}{ Week } & \multicolumn{2}{|c|}{ Blood Glucose, $\mathbf{m M} \pm$ SEM } & \multicolumn{2}{|c|}{ IOP, $\mathbf{m m ~ H g ~} \pm$ SEM } & \multirow[b]{2}{*}{$\mathbf{B P}$} & \multicolumn{2}{|c|}{$\mathrm{SBP}, \mathrm{mm} \mathrm{Hg} \pm \mathrm{SEM}$} \\
\hline & Citrate & STZ-Diabetes & Citrate & STZ-Diabetes & & Citrate & STZ-Diabetes \\
\hline 1 & $5.8 \pm 0.2$ & $23.1 \pm 0.9^{*}$ & $13.4 \pm 0.4$ & $13.4 \pm 0.5$ & 1 & $110.2 \pm 7.6$ & $110.3 \pm 4.2$ \\
\hline 2 & $5.3 \pm 0.2$ & $21.6 \pm 1.0^{*}$ & & & 2 & $92.0 \pm 5.5$ & $102.7 \pm 3.9$ \\
\hline
\end{tabular}

* Denotes significance $(P<0.001)$ between citrate and STZ-diabetic animals for blood glucose $(\mathrm{mM})$.

induced in 6-week-old male Long-Evans rats (Monash Animal Services, Clayton, Victoria, Australia) via a tail vein injection of $65 \mathrm{~kg} / \mathrm{mg} \mathrm{STZ}$ (MP Biomedicals, Seven Hills, New South Wales, Australia) dissolved in 0.1 M sodium citrate buffer (pH 4.5; Sigma-Aldrich Pty Ltd., Castle Hill, New South Wales, Australia). Animals were fasted overnight $(\sim 12$ hours) prior to STZ-injection. Control animals were given tail vein injections of $0.1 \mathrm{M}$ sodium citrate buffer (citrate group). One week following STZ-injection, blood glucose (Table 1) was measured (Ascensia Esprit Glucometer; Bayer Australia, Ltd., Pymble, New South Wales, Australia) to confirm that all STZ-treated animals had blood glucose higher than $15 \mathrm{mM}$. To prevent ketoacidosis and excessive loss of body weight, 2 units of insulin was administered each day for 2 weeks. After 2 weeks, only those animals with blood glucose higher than $20 \mathrm{mM}$ continued to receive insulin injection ( 2 units/d). Animals were housed in a $22^{\circ} \mathrm{C}$ environment with a 12:12-hour light-dark cycle (maximum 40 lux), with normal rat chow (Barastoc rat chow; Ridley AgriProducts, Melbourne, Victoria, Australia) and water available ad libitum.

Electrophysiology and blood flow measurement were conducted under general anesthesia (ketamine-xylazine, $60: 5 \mathrm{mg} / \mathrm{kg}$, intramuscularly [IM]). It is worth noting that ketamine-xylazine anesthesia may have neuroprotective effects ${ }^{19}$; however, our low dose of ketamine should provide similar effects in both citrate control and STZ-injected animals. A topical anesthetic ( $0.5 \%$ proxymetacaine hydrochloride, eye drops, Alcaine, $5 \mathrm{mg} / \mathrm{mL}$; Alcon Laboratories, Frenchs Forest, New South Wales, Australia) and a mydriatic (0.5\% tropicamide, Mydriacyl, 5 $\mathrm{mg} / \mathrm{mL}$; Alcon Laboratories) were also given. A circulating water heat pad was used to maintain body temperature at approximately $37^{\circ} \mathrm{C}$.

\section{Acute IOP Elevation}

Baseline IOP was obtained from each animal using a noninvasive applanation tonometer (Tonopen XL; Medtronic Solan, Jacksonville, FL) using light sedation ( $50 \%$ dose, ketamine-xylazine, $30 \mathrm{mg} / \mathrm{kg}: 2.5$ $\mathrm{mg} / \mathrm{kg}$, IM). This established that STZ treatment did not affect baseline IOP (Table 1). To ensure that any functional and ocular blood flow susceptibility is independent of ocular perfusion pressure (blood pressure - IOP), systolic blood pressure (SBP, Table 1) was assayed via noninvasive tail cuff sphygmomanometer (ML125; ADInstruments Pty, Ltd., Bella Vista, New South Wales, Australia). To assess ocular susceptibility to stress, one randomly selected eye underwent manometric IOP elevation from 10 to $100 \mathrm{~mm} \mathrm{Hg}$ in increments of 5 $\mathrm{mm} \mathrm{Hg}$ (3 minutes per step) using a height-adjustable reservoir (Hanks balanced salt solution; Sigma-Aldrich Pty Ltd.) attached to a 30-gauge cannula via polyethylene tubing. For electroretinogram (ERG) assessment, the cannula was inserted into the vitreal chamber to allow for placement of ERG electrodes. In those animals undergoing blood-flow studies, a laser Doppler flowmetry (LDF) (ML191; ADInstruments Pty, Ltd.) needle probe (MNP110XP; ADInstruments Pty, Ltd.) was inserted into the vitreal chamber and, thus, IOP was manometrically controlled via an anterior chamber cannula. Previously, we have shown that the pressure between the anterior and vitreal chamber quickly equilibrates regardless of the location of the cannula. ${ }^{20}$

\section{Electroretinography}

Prior to functional measurements, all animals were dark-adapted overnight (approximately 12 hours) and procedures were conducted in the dark with the aid of night-vision goggles (NVMT24021; Yukon Advanced Optics, Sellmark Corporation, Mansfield, TX) and an infrared light (IR) source ( $\lambda=880 \mathrm{~nm}, 18$-LED IR spotlight; Jaycar, Rydalmere, New South Wales, Australia) adapted onto a dissecting microscope (Zeiss OPMI-1 Lab; Carl Zeiss Pty, Ltd., Jena, Germany).

Baseline ERG was measured 3 weeks following STZ or citrate treatment. Responses were measured across a range of calibrated (IL1700; International Light Technologies, Inc., Newbury Port, MA) luminous energies ( -6.79 to $2.07 \mathrm{log}$ cd.s. $\mathrm{m}^{-2}$ ) delivered via a Ganzfeld integrating sphere $(170 \mathrm{~mm}$ diameter; Photometric Solutions International, Huntingdale, Victoria, Australia). This range returns measures of photoreceptoral (a-wave) ${ }^{21}$ and ON-bipolar cell (b-wave). ${ }^{22}$ The dimmest flashes elicit the scotopic threshold response (STR), which provides information about the integrity of retinal ganglion cells. ${ }^{23-25}$

To quantify functional susceptibility to acute IOP challenge, ERGs were recorded 4 weeks following citrate or STZ injection (1 week after baseline assessment). Two luminous energies were assayed at each step of IOP elevation. The first luminous energy was chosen to elicit an ERG dominated by a rod-driven b-wave $\left(-1.42 \log\right.$ cd.s.m $\left.{ }^{-2}\right)$, which has been widely used to assay the effects of ischemia and IOP stress. ${ }^{20,26,27}$ The second, much dimmer, luminous energy $\left(-5.25 \log \mathrm{cd} . \mathrm{s} . \mathrm{m}^{-2}\right)$ was chosen to elicit a retinal ganglion cell-dominated STR, a component of the ERG known to be particularly sensitive to IOP elevation.

ERGs were recorded using custom-made corneal (active) and scleral (reference, ring-shaped) chlorided silver electrodes (99.99\% purity, 0.329 mm, 29-gauge; A\&E Metal Merchants, Sydney, New South Wales, Australia). Both electrodes from each eye were grounded to a stainless steel electrode (F-E2-30; Grass Telefactor, West Warwick, RI) inserted subcutaneously into the tail. Eyes were lubricated after electrode placement and periodically throughout the session with $1.0 \%$ carboxymethylcellulose sodium (Celluvisc; Allergan, Irvine, CA). Signals were digitized at $4 \mathrm{kHz}$ (ML785, Powerlab/8SP, 1000× amplification; ADInstruments Pty, Ltd.), with band pass settings of -3 $\mathrm{dB}$ at 0.3 to $1000 \mathrm{~Hz}$.

\section{Ocular Blood Flow: Laser Doppler Flowmetry}

Ocular blood flow was measured using LDF (ML191; ADInstruments Pty, Ltd.) as has been used previously. ${ }^{28-30}$ This approach provides realtime continuous measurement of tissue microvascular perfusion. In the eye, LDF reflects both retinal and choroidal blood flow, ${ }^{27}$ thus the term "ocular blood flow" (OBF) is used throughout the study.

The LDF needle probe $(0.48 \mathrm{~mm}$ outer diameter $\times 25 \mathrm{~mm}$ length; MNP110XP; ADInstruments Pty, Ltd.) was inserted $2 \mathrm{~mm}$ behind the limbus, into the vitreal chamber. Both the LDF probe and IOP cannula (anterior chamber) were stabilized and positioned with micromanipulators (MM-3; Narishige Co., Ltd., Tokyo, Japan).

\section{Retinal Tissue Isolation and Real-Time PCR}

On completion of in vivo experiments, eyes were enucleated and animals were euthanized $(0.3 \mathrm{~mL}$ pentobarbitone sodium, Lethabarb; Virbac Pty, Ltd., Peakhurst, New South Wales, Australia). Retinas were excised, snap frozen in liquid nitrogen, and stored at $-80^{\circ} \mathrm{C}$ for RNA isolation. Real-time quantitative PCR was conducted, for control and IOP-treated eyes in both citrate and STZ-treated groups, to quantify mRNA expression for specific target genes of interest (nNos, eNos, and $i$ Nos) relative to a housekeeping gene (hypoxanthine phosphorylribo- 
Table 2. Oligonucleotide Primers for Nitric Oxide Synthase Isoenzymes (eNos, iNos, and nNos) and the Housekeeper Gene, HPRT

\begin{tabular}{|c|c|c|c|c|}
\hline Gene & $\begin{array}{l}\text { Accession No. } \\
\text { (PubMed.org) }\end{array}$ & Primer Sequences & Source Organism & Amplicon Size, bp \\
\hline eNos & NM_021838.2 ${ }^{60}$ & $\begin{array}{l}\text { F: 5'-TGGCAGCCCTAAGACCTATG-3' } \\
\text { R: 5'-AGTCCGAAAATGTCCTCGTG-3' }\end{array}$ & Rattus norvegicus (Norway rat) & 243 \\
\hline iNos & NM_012611.3 $3^{60}$ & $\begin{array}{l}\text { F: 5'-CCTTGTTCAGCTACGCCTTC-3' } \\
\text { R: 5'-GGTATGCCCGAGTTCTTTCA-3' }\end{array}$ & Rattus norvegicus (Norway rat) & 179 \\
\hline nNos & NM_052799.161 & $\begin{array}{l}\text { F: 5'-CCTTCCGAAGCTTCTGGCAACAGC-3' } \\
\text { R: 5'-TGGACTCAGATCTAAGGCGGTTGG-3' }\end{array}$ & Rattus norvegicus (Norway rat) & 474 \\
\hline$H P R T$ & NM_000194.2 $2^{62}$ & $\begin{array}{l}\text { F: 5'-GGAGGCCATCACATTGTAGC-3' } \\
\text { R: 5'-CCACAATCAAGACATTCTTTC-3' }\end{array}$ & Homo sapiens (Human) & 229 \\
\hline
\end{tabular}

F, forward; R, reverse.

syl transferase $[H P R T])$. For oligonucleotide primer sequences, see Table 2.

RNA was isolated from dissected retinal tissue using a commercially available kit (RNeasy Mini Kit; Qiagen Pty, Ltd., Doncaster, Victoria, Australia). Isolated retinas were homogenized and treated with DNase-I to eliminate possible contamination by genomic DNA. The purified RNA ( $10 \times$ dilution with RNase-free $\left.\mathrm{dH}_{2} \mathrm{O}\right)$ was then quantified using a spectrophotometer (UV-2550; Shimadzu Corporation, Tokyo, Japan). RNA concentration was determined by the absorbance at $260 \mathrm{~nm}$ (RNeasy Mini Handbook for RNeasy Mini Kit, 2006; Qiagen Pty, Ltd.).

A 500-ng volume of purified RNA was transcribed to CDNA via reverse transcription (RT) reaction. An Omniscript RT Kit (Qiagen Pty, Ltd.) was used in combination with $0.5 \mu \mathrm{M}$ oligo $\mathrm{d}(\mathrm{T})$ primers to synthesize cDNA. These samples were incubated at $37^{\circ} \mathrm{C}$ for 1 hour, followed by dilution in $\mathrm{dH}_{2} \mathrm{O}$ into $5 \mathrm{ng} / \mu \mathrm{L}$ aliquots and stored at $-20^{\circ} \mathrm{C}$. This was followed by RT (M-MLV Reverse Transcriptase, $200 \mu / \mu \mathrm{L}$ and Random Hexamers, $20 \mu \mathrm{g}$; Promega Corporation, Alexandria, New South Wales, Australia).

The resultant cDNA was amplified by using real-time PCR (LightCycler 3 Carousel-based system; Roche Diagnostics Australia Pty, Ltd., Castle Hill, New South Wales, Australia). The cDNA sample $(1 \mu \mathrm{L})$ in $\mathrm{dH}_{2} \mathrm{O}$ was combined with a master mix containing magnesium chloride $\left(\mathrm{MgCl}_{2}\right)$, reverse and forward primers (Sigma-Aldrich Pty, Ltd.) and a reaction medium (FastStart DNA Master SYBR Green I; Roche Diagnostics Australia Pty, Ltd.). To quantify the amount of gene transcript, an external standard (the respective PCR-amplified cDNA products) was serially diluted to produce a four-point standard curve. A gene copy number was calculated for each dilution for post hoc analysis. All real-time PCR samples were amplified in sets of triplicates to minimize handling error. PCR results were analyzed using LightCycler Data Analysis software (v 3.5.28; Roche Molecular Diagnostics, Pleasanton, CA).

\section{Data Analysis}

Photoreceptoral Response. The leading edge of the scotopic awave (PIII) was modeled (Equation 1), ${ }^{31}$ returning a measure of photoreceptoral function. The summed rod photoreceptor response (PIII) as a function of time ( $t$, seconds) and luminous energy $(i, \log$ cd.s. $\left.\mathrm{m}^{-2}\right)$, given by a maximal amplitude $\left(R m_{P I I}, \mu \mathrm{V}\right)$, a sensitivity $(S$, $\left.\log \left[\mathrm{m}^{2} \cdot \mathrm{cd}^{-1} \cdot \mathrm{s}^{-3}\right]\right)$, and a brief delay $\left(t_{d}\right.$, seconds; largely recording equipment delay),

$$
P I I I(i, t)=R m_{P I I I} \cdot\left[1-\exp \left(-i \cdot s \cdot\left(t-t_{d}\right)^{2}\right)\right], \quad t>t_{d} .
$$

This function was modeled over an ensemble of the 2 brightest luminous energies (1.95 and $2.07 \mathrm{log}$ cd.s. $\mathrm{m}^{-2}$ ). The delay $\left(t_{d}\right)$ was 4.07 \pm 0.05 and $4.15 \pm 0.03 \mathrm{~ms}$ for citrate and STZ-treated groups, respectively. These $t_{d}$ values were then fixed; thus, only $S$ and $R m_{P I I I}$ were allowed to float in the final model, which was optimized by minimizing the sum-of-squares merit function using the Excel Solver module (Microsoft, Redmond, WA).

Rod and Cone ON-Bipolar Cell Responses. To isolate the ONbipolar cell component of the ERG (PII), the modeled PIII was subtracted from the raw waveform. ${ }^{32}$ PII amplitudes measured at a fixed time of $70 \mathrm{~ms}$ were plotted as a function of luminous energy and modeled using a saturating hyperbolic function, as given in Equation 2. The amplitude $(V, \mu \mathrm{V})$ as a function of luminous energy $(i$, $\log$ cd.s. $\left.\mathrm{m}^{-2}\right)$ is given by a maximal amplitude $\left(V_{\max }, \mu \mathrm{V}\right)$, semisaturation constant $\left(k, \log\right.$ cd.s. $\mathrm{m}^{-2}$ ), where sensitivity is $1 / k$ $\left(\mathrm{cd}^{-1} \cdot \mathrm{s}^{-1} \cdot \mathrm{m}^{2}\right)$ and $n$ is a dimensionless exponent that determines the slope of the function.

$$
V(i)=V_{\max } \frac{i^{n}}{i^{n}+k^{n}}
$$

The dark-adapted ERG reflects contributions from both rod and cone pathways. Therefore, Equation 2 was fit to luminous energies below those containing cone responses. This was determined by establishing the amplitude of the "putative" rod response from a twin-flash paradigm at $2.07 \mathrm{log}$ cd.s.m ${ }^{-2}$. By using a 500-ms interstimulus interval, the long refractory period of the rod system means that only cone responses are recorded in the second flash, which is then subtracted from the mixed response to return the putative rod response. Group data for rod amplitude (at $2.07 \log$ cd.s.m ${ }^{-2}$ ) was compared with the amplitude of mixed responses for all other luminous energies. Amplitudes for luminous energies greater than $-0.82 \log$ cd.s. $\mathrm{m}^{-2}$ were found to be significantly larger than the isolated rod PII amplitude $(P<0.05)$, indicating significant cone contribution. Thus, Equation 2 was fit to luminous energies between -6.79 to $-0.82 \log$ cd.s. $\mathrm{m}^{-2}$, as well as the isolated rod PII at $2.07 \mathrm{log}$ cd.s. $\mathrm{m}^{-2}$ by minimizing the sum-of-squares merit function using the Excel Solver module (Microsoft).

Scotopic Threshold Response. Amplitudes of the STR were evaluated for luminous energies between -6.79 and $-5.25 \mathrm{log}$ cd.s. $\mathrm{m}^{-2}$ by taking the amplitude at a fixed time of 110 and $220 \mathrm{~ms}$ to return positive (pSTR) and negative (nSTR) components, respectively.

Susceptibility to IOP Elevation. The change in ERG or ocular blood flow at each IOP level was expressed as a percentage relative to responses collected at baseline IOP of $10 \mathrm{~mm} \mathrm{Hg}$ (stress/baseline, \%). As previous, ${ }^{20}$ an inverse cumulative normal function (Equation $3^{20}$ ) was used to paramatize the relationship between relative (mean \pm SEM) ERG amplitudes as a function of IOP. This function was applied assuming a maximum of $100 \%$, to return best-fit parameters for the mean $(\mu)$ and $1 /$ slope $(\sigma)$. The mean represents the IOP level at which the response has been reduced to $50 \%\left(\mathrm{IOP}_{50}\right)$ and $1 /$ slope represents the steepness of the curve.

$$
f(x)=1-\frac{1}{\sqrt{2 \pi \sigma}} e^{-\left[\frac{(x-\mu)^{2}}{2 \sigma^{2}}\right]}
$$




\section{Statistics}

To compare blood glucose, baseline IOP, SBP, and ERG, as well as changes in Nos expression, the following statistics were employed. A Kolmogorov-Smirnov normality test and Barlett's test for equal variance were performed before comparing treatments. Data showing normality and equal variances were analyzed using parametric analysis, such as unpaired or paired $t$-test, repeated measures ANOVA (RM-ANOVA), oneway ANOVA, and two-way ANOVA. For one-way ANOVA analysis, a Dunnett's post hoc test was applied to determine significant departures from baseline. For data failing criteria for parametric statistics, two approaches were used. First, for comparisons involving multiple factors (treatment and IOP), a more conservative $\alpha$-value of 0.035 was used to protect against false positives. Second, for comparison of a single factor between STZ-diabetic and citrate animals, a nonparametric Mann-Whitney test was adopted.

As previous, ${ }^{20}$ to compare the susceptibility of citrate and STZdiabetic ERG and blood flow to IOP elevation, a bootstrap method was used to generate estimates of the mean and $95 \%$ confidence limits (CLs) for $\operatorname{IOP}_{50}(\mu)$ and $1 /$ slope $(\sigma)$. This approach has the advantage that it does not make any assumptions regarding the underlying distribution of the data and provides a more robust estimate of parameter variability in transition functions, such as in Equation 3. ${ }^{33,34}$ As previous, ${ }^{35}$ each iterative bootstrap sample consisted of $n$ random selections taken (with replacement) from each group. The bootstrap sample was then modeled as a function of IOP using Equation 3, to return $\mathrm{IOP}_{50}$ and 1/ slope for this particular sample. After 1000 iterations, the 25th and 97th percentiles were taken as the "bootstrap" bounds of the 95\% CL. This approach allows a robust comparison of the relative sensitivity of various citrate and STZ-diabetic eyes to IOP elevation. When the best-fit parameter of the STZ-diabetic group falls outside the 95\% CL of the respective parameter for the citrate group, then $P$ is less than 0.05 .

\section{Results}

Table 1 shows that at $1(P<0.001)$ and 2 weeks following STZ injection $(P<0.001)$, there was significant elevation of blood glucose in STZ-treated animals compared with citrate controls. It has been reported that systemic hyperglycemia can modify IOP and blood pressure, ${ }^{36}$ which may modify ocular perfusion pressure (OPP), an important determinant of ocular blood flow. Noninvasive measurement of baseline IOP revealed no significant difference between citrate and diabetic groups $(P=$ 0.932; Table 1). SBP was similar when measured noninvasively at the beginning (citrate versus STZ, $P=0.991 ; \mathrm{BP} 1$ ) and end of the IOP challenge protocol $(P=0.116 ; \mathrm{BP} 2)$. Although there was a small decrease in SBP from the beginning to the end of the experimental protocol, this was not statistically significant for citrate $(P=0.073)$ and STZ-diabetic animals $(P=0.325)$.

\section{Baseline ERG: STR and b-Wave}

Figure 1 shows retinal function across a range of luminous energies recorded at baseline and 3 weeks following citrate (thin traces) or STZ (thick traces) injection. Three weeks of hyperglycemia had little obvious effect on the ERG in citrate and STZ-diabetic animals.

Saturated phototransduction amplitude $\left(\mathrm{Rm}_{\mathrm{PIII}}\right.$, Fig. $\left.2 \mathrm{~A}\right)$ and sensitivity (S) (Fig. 2B) were not significantly different between citrate and diabetic eyes $\left(\mathrm{Rm}_{\mathrm{PIII}}: P=0.70, \mathrm{~S}: P=0.76\right)$. The ERG PII (reflecting ON-bipolar cell function) was assessed by fitting the luminous energy-response function for amplitudes taken at a fixed time of $70 \mathrm{~ms}$ with a saturating hyperbolic function (Equation 2). The saturated ON-bipolar cell amplitude was not significantly different between STZ-diabetic and citrate eyes $\left(\mathrm{V}_{\max }, P=0.657\right.$; Fig. 2C). However, there was a significant reduction in the PII sensitivity in the STZ group when compared with citrate controls $(1 / k: P=0.006 ;$ Fig. $2 \mathrm{D})$.

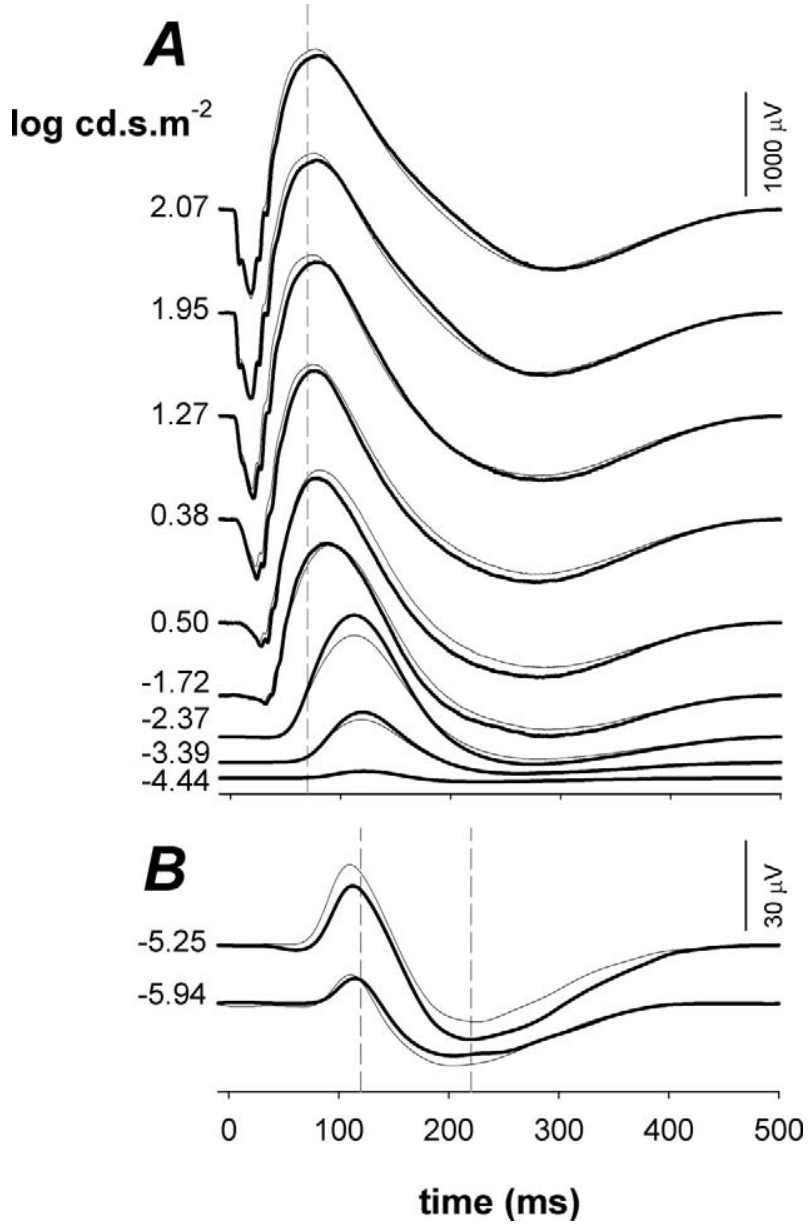

Figure 1. Comparison of retinal function between normal and diabetic groups at 3 weeks post STZ-induction. (A) Representative dark-adapted ERG waveforms for normal (thin trace) and diabetic (thick trace) groups elicited across a wide range of luminous energies ( -4.44 to $2.07 \mathrm{log}$ cd.s. $\mathrm{m}^{-2}$ ). (B) Representative scotopic threshold responses elicited at dim light levels $\left(-6.79\right.$ to $-5.25 \log$ cd.s.m $\left.{ }^{-2}\right)$.

The retinal ganglion cell response as given by STR amplitudes at a fixed time of $110 \mathrm{~ms}$ (Fig. 2E) was significantly reduced in the diabetic group $(P<0.001)$. On the other hand, the amplitude at $220 \mathrm{~ms}$ was larger in the STZ group $(P=0.024$; Fig. $2 \mathrm{~F})$. The STZ-treated group also showed a delay in the pSTR $(P=0.016$; Fig. $2 \mathrm{G})$; however, nSTR implicit time $(P=0.847$; Fig. $2 \mathrm{H})$ was similar to citrate control eyes.

\section{Effect of Acute IOP on Retinal Function in STZ- Induced Diabetes}

Figure 3 shows that both the ERG b-wave (Fig. 3A) and STR (Fig. 3B) responses in STZ-diabetic eyes are more sensitive to IOP elevation than the citrate group. Quantification using an inverse cumulative normal function confirmed that STR and bwave amplitudes in STZ-diabetic eyes were significantly more susceptible ( $\mu$, IOP at $50 \%$ response reduction, $\mathrm{IOP}_{50}$ ) to IOP elevation, as summarized in Table 3. Specifically, the STZdiabetic b-wave IOP-response function was $4.8 \mathrm{~mm} \mathrm{Hg}(-7.7 \%)$ more susceptible $\left(\mathrm{IOP}_{50}: 60.3 \mathrm{~mm} \mathrm{Hg}\right.$, confidence interval $[\mathrm{CI}]_{2.5-97.5}: 58.0-62.8 \mathrm{~mm} \mathrm{Hg}$; Fig. $\left.4 \mathrm{~A}\right)$ than the citrate group $\left(\mathrm{IOP}_{50}\right.$ : $65.1 \mathrm{~mm} \mathrm{Hg} \mathrm{CI} 2.5-97.5$ : 61.9-68.6 mm Hg). The STR in 

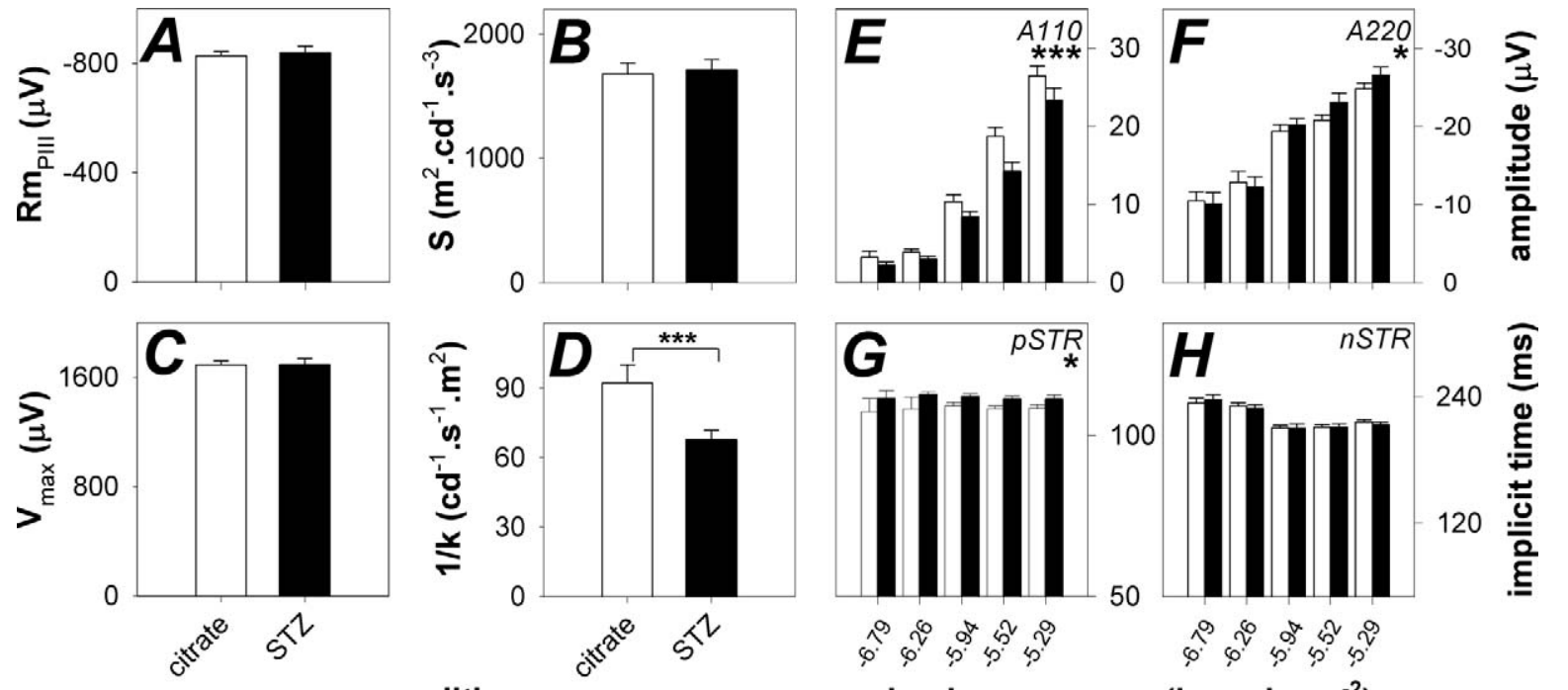

condition

luminous energy $\left(\log\right.$ cd.s. $\left.\mathrm{m}^{-2}\right)$

Figure 2. Comparison of parameters reflecting retinal cell function for normal ( $n=14$, unfilled bars/symbols) and diabetic eyes $(n=20$, filled bars/symbols). Averaged ( \pm SEM) phototransduction saturated amplitude $(\mathbf{A}) \mathrm{Rm}_{\mathrm{PIII}}(\mu \mathrm{V})$ and $(\mathbf{B})$ sensitivity $\left(\mathrm{S}, \mathrm{m}^{2} \cdot \mathrm{cd}^{-1} \cdot \mathrm{s}^{-3}\right)$. These parameters are derived from an ensemble fit of the ERG a-wave elicited at 1.95 and $2.07 \log$ cd.s.m ${ }^{-2}$ (Equation 1). ON-bipolar cell function parameters (C) maximal

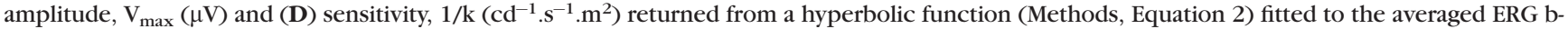
wave amplitudes taken at a fixed time of $70 \mathrm{~ms}$, across a range of luminous energies ( -6.79 to $\left.2.07 \mathrm{log} \mathrm{cd} . \mathrm{s} . \mathrm{m}^{-2}\right)$, anchored to the "putative" rod ON-bipolar cells response ( $\left.2.07 \mathrm{log} \mathrm{cd} . \mathrm{s} . \mathrm{m}^{-2}\right)$. Averaged inner retinal function $(\mu \mathrm{V}$, scotopic threshold response, STR) measured at fixed times of (E) 110 and (F) $220 \mathrm{~ms}$. Implicit times of (G) peak (pSTR) and (H) trough (nSTR) amplitudes of STR ( -6.79 to $-5.25 \log$ cd.s.m $^{-2}$ ). Asterisks denote significance $\left({ }^{*} P<0.05,{ }^{* * *} P<0.001\right)$

STZ animals was $7.4 \mathrm{~mm} \mathrm{Hg}(-11.6 \%)$ more susceptible $\left(\mathrm{IOP}_{50}\right.$ : $60.1 \mathrm{~mm} \mathrm{Hg}, \mathrm{CI}_{2.5-97.5}: 57.0-62.0 \mathrm{~mm} \mathrm{Hg}$ ) in STZ-diabetic animals than the citrate group ( $\mathrm{IOP}_{50}: 67.5, \mathrm{CI}_{2.5-97.5}: 62.1-$ $72.4 \mathrm{~mm} \mathrm{Hg}$; Table 3). No difference was found between the slope of the inverse cumulative normal function for the b-wave or STR (Table 3).

\section{Effect of Acute IOP Elevation on Ocular Blood Flow in STZ-Diabetes}

To consider whether the increased functional susceptibility observed in STZ-diabetic eyes was mediated by an impaired blood flow response to stress, ocular blood flow was measured in response to the same IOP challenge.

Similar to retinal function, ocular blood flow (Fig. 4A) in STZ-diabetic eyes was almost $10 \mathrm{~mm} \mathrm{Hg}$ more susceptible to acute IOP challenge than the citrate group (STZ, IOP 50 : 43.9, $\mathrm{CI}_{2.5-97.5}$ : $40.8-46.8 \mathrm{~mm} \mathrm{Hg}$, filled symbols versus citrate, $\mathrm{IOP}_{50}, 53.4, \mathrm{CI}_{2.5-97.5}: 50.7-56.1 \mathrm{~mm} \mathrm{Hg}$, unfilled symbols; Table 3 ). Table 3 also shows that no change was found in the spread of the function (or sensitivity) between the STZ-diabetic $\left(\sigma, 24.0, \mathrm{CI}_{2.5-97.5}: 20.6-27.1\right)$ and the citrate group $(\sigma, 26.1$, $\left.\mathrm{CI}_{2.5-97.5}: 21.9-30.0\right)$.

Figure $4 \mathrm{~B}$ examines the relationship between retinal function and ocular blood flow by taking the difference between the STR and blood flow. These data show that for IOPs below $80 \mathrm{~mm} \mathrm{Hg}$, blood flow is more affected than the STR amplitude. Comparison between diabetic and citrate groups suggests that for IOPs between 55 and $100 \mathrm{~mm} \mathrm{Hg}$ $(P<0.001)$, the gap between the STR and blood flow was smaller in STZ-diabetic eyes than citrate control eyes. This finding suggests that the relationship between blood flow

TABLE 3. Inverse Cumulative Normal Function Parameters (Susceptibility, $\mu$, and Spread, $\sigma$ ) for Citrate and STZ-Diabetic Eyes

\begin{tabular}{|c|c|c|c|c|c|}
\hline & \multicolumn{5}{|c|}{ Inverse Cumulative Normal Function } \\
\hline & \multicolumn{3}{|c|}{ Susceptibility, Functional $\mathrm{IOP}_{50}, \mu, \mathrm{mm} \mathbf{H g}$} & \multicolumn{2}{|r|}{ 1/Slope, $\sigma$} \\
\hline & Best Fit, $\mathrm{IOP}_{50}, \mathrm{~mm} \mathrm{Hg}$ & Difference & $95 \%$ CI, 2.5 to $97.5 \%$ & Best Fit & $95 \%$ CI, 2.5 to $97.5 \%$ \\
\hline \multicolumn{6}{|l|}{ ERG } \\
\hline Citrate b-wave & 65.1 & & 61.9 to 68.6 & 11.8 & 8.1 to 14.1 \\
\hline STZ b-wave & $60.3^{*}$ & $4.8(-7.7 \%)$ & 58.0 to 62.8 & 10.4 & 7.4 to 12.5 \\
\hline Citrate STR & 67.5 & & 62.1 to 72.4 & 12.0 & 5.0 to 15.1 \\
\hline STZ STR & $60.1 \dagger$ & $7.4(-11.6 \%)$ & 57.0 to 62.0 & 10.4 & 5.4 to 12.3 \\
\hline \multicolumn{6}{|l|}{ OBF } \\
\hline Citrate & 53.4 & & 50.7 to 56.1 & 26.1 & 21.9 to 30.0 \\
\hline STZ & $43.9 \ddagger$ & & 40.8 to 46.8 & 24.0 & 20.6 to 27.1 \\
\hline
\end{tabular}

\footnotetext{
* $P=0.0038$ compared with the lower limit citrate controls.

$\dagger P=0.016$ compared with the lower limit citrate controls

$\ddagger P<0.001$ compared with the lower limit citrate control.
} 


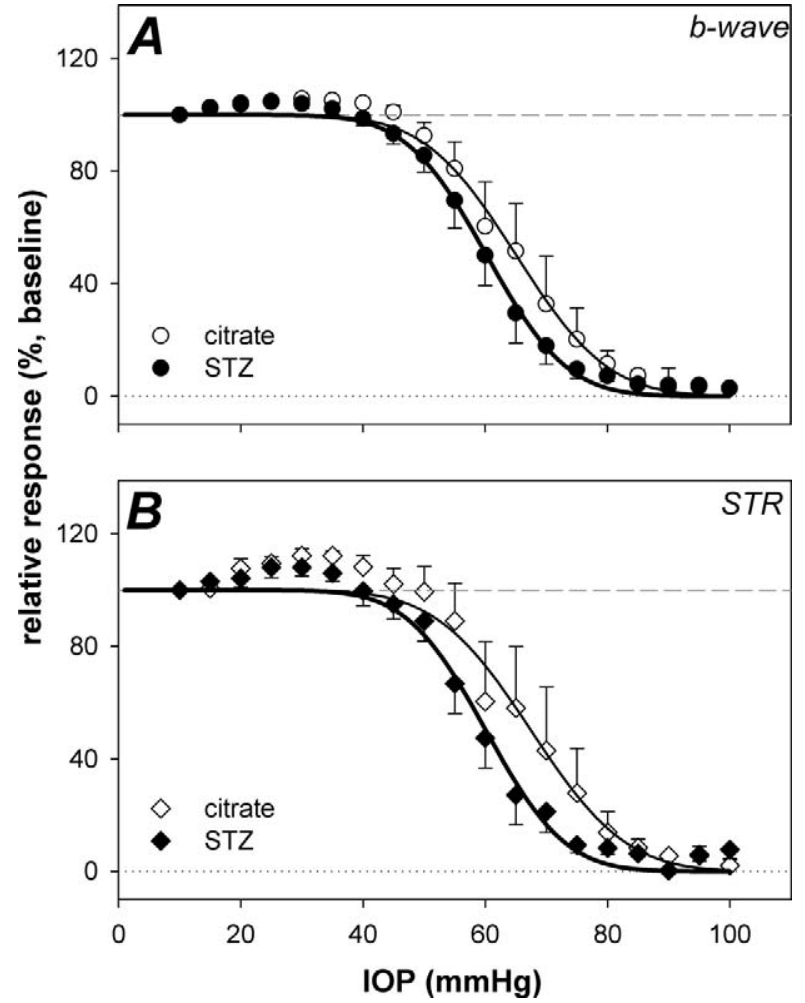

Figure 3. Comparison of relative (A) b-wave (ON-bipolar cells) and (B) STR (inner retina) averaged ( \pm SEM) amplitudes as a function of IOP in citrate (unfilled symbols) and diabetic groups (filled symbols). IOPresponse relationships were described with use of an inverse cumulative normal function (equation 3; citrate, thin curve, versus diabetic, thick curve) to quantify change in amplitude as a function of IOP. This function returns a best-fit $\mu$ and $\sigma$, which represents sensitivity and spread, respectively. CIs for these parameters were derived from a nonparametric bootstrap method (Table 3). Dotted and dashed lines denote $0 \%$ and $100 \%$ relative amplitude, respectively.

and retinal function during stress is altered in STZ-diabetic eyes.

\section{Effect of STZ-Diabetes and Acute IOP on NOS mRNA Expression}

Abnormal levels of Nos isoenzymes (eNos, iNos, and nNos) are thought to contribute to vascular, neuronal, and glial dysfunction. ${ }^{37-40}$ Thus, we considered if the increased vulnerability to IOP challenge observed in STZ-diabetic eyes was associated with changes in nitric oxide synthase activity. Combining both control and IOP eyes, the STZ-diabetic group (Fig. 5A) for mRNA expressions for eNos (citrate: 17.4 \pm 4.8 versus STZ: $13.3 \pm 3.9 ; P=0.153$ ), $n$ Nos (citrate: $7.2 \pm 2.2$ versus STZ: $7.2 \pm 3.1\left[\times 10^{3}\right.$ copies $\left.] ; P=0.463\right)$, and $i$ Nos (citrate: $0.6 \pm 0.2$ versus STZ: $1.2 \pm 0.7\left[\times 10^{2}\right.$ copies]; $P=$ 0.640) was not significantly different between diabetic and citrate eyes. Interestingly, IOP challenge on mRNA expression (\%, Fig. 5B) increased eNos mRNA expression by $49.7 \%$ in citrate control retinas, an effect significantly greater than the STZ-diabetic group $(-5.7 \%, P=0.034)$. However, no significant difference was found for $n$ Nos $(P=0.789)$ and $i$ Nos $(P=0.859)$ expression between citrate and STZ-diabetic groups. In addition, the IOP-induced change in eNos mRNA expression was significantly correlated with the susceptibility of ocular blood flow to IOP challenge $\left(\mathrm{IOP}_{50}, y=0.131 x+\right.$
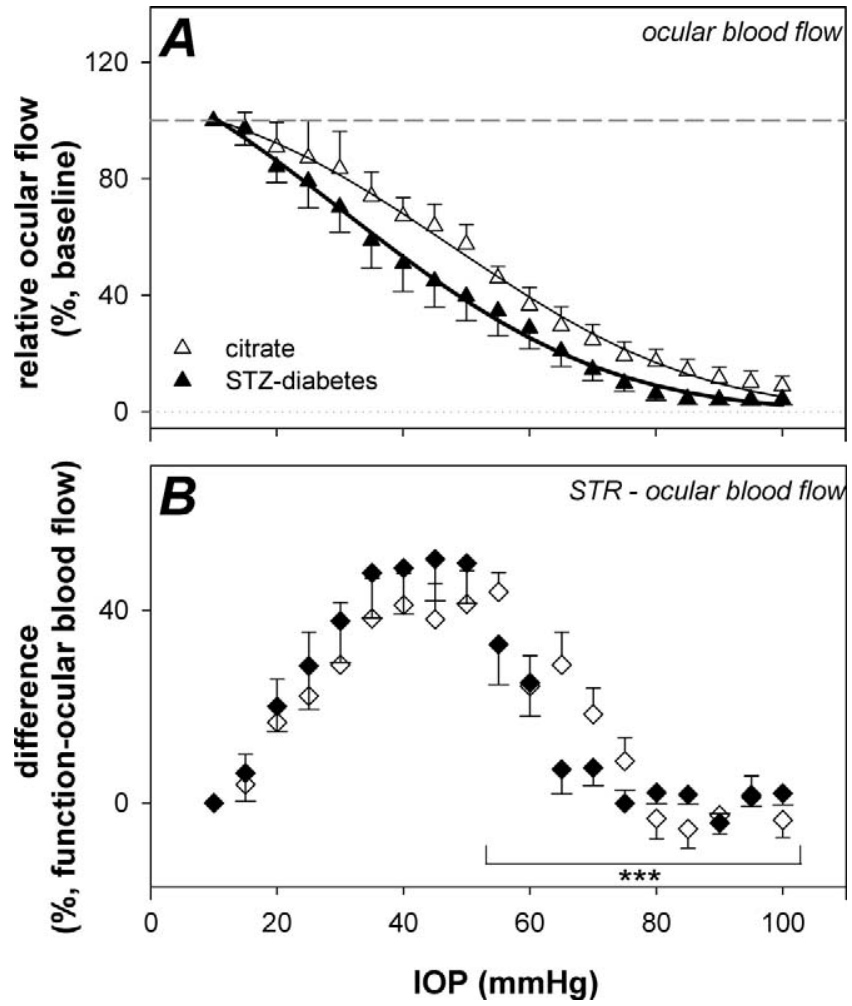

Figure 4. Effect of acute IOP challenge on ocular blood flow in normal ( $n=8$, unfilled symbols) and diabetic animals ( $n=10$, filled symbols). (A) Averaged ocular blood flow ( \pm SEM) relative to each eye's own baseline (100\%, dasbed line) for normal and diabetic groups. Each curve was modeled with an inverse cumulative normal function (equation 3) to quantify the effect between treatment groups. CIs for these parameters were derived from a nonparametric bootstrap method (Table 3). Dotted and dashed lines denote $0 \%$ and 100\% relative amplitude, respectively. (B) Difference (\%) between relative inner retinal function and ocular blood flow responses across increasing IOP. Asterisks denote significance $\left({ }^{* * *} P<0.001\right)$.

46.8, $r_{\mathrm{s}}=0.649$, Spearman correlation, $r_{\mathrm{s}}=0.649, P<0.05$, Fig. 5C).

\section{Discussion}

The key finding of this study was that as early as 4 weeks following the onset of chronic hyperglycemia, before changes in the major components of the ERG, ${ }^{18,41-43}$ retinal function (Fig. 3) shows greater susceptibility to acute IOP challenge. Although this is consistent with the work of Kanamori et al., ${ }^{17}$ it is inconsistent with the study of Casson and colleagues. ${ }^{26}$ These authors reported that 5 days or 6 weeks post-STZ injection $(60 \mathrm{~kg} / \mathrm{mg}$; blood glucose: approximately $25 \pm 3.2$ $\mathrm{mM}$ ), b-wave recovery at 3 to 7 days following IOP elevation to ischemic level $(110 \mathrm{~mm} \mathrm{Hg})$ was better in diabetic eyes. It may be that hyperglycemia is protective against ischemic levels of IOP elevation of $110 \mathrm{~mm} \mathrm{Hg}$ (as long as 60 minutes). Indeed, a "protective" effect of hyperglycemia may be important for neuronal viability under acute anaerobic stress. ${ }^{44}$ The IOP used in this study only reached $100 \mathrm{~mm}$ $\mathrm{Hg}$ for 3 minutes.

The increase in ERG susceptibility to IOP elevation was associated with an increase in susceptibility in ocular blood flow (Figs. 3B, 4A). Indeed, ocular blood flow in diabetic eyes was $10 \mathrm{~mm} \mathrm{Hg}$ more sensitive to IOP elevation than in the citrate group (Fig. 4), which is somewhat similar to the 

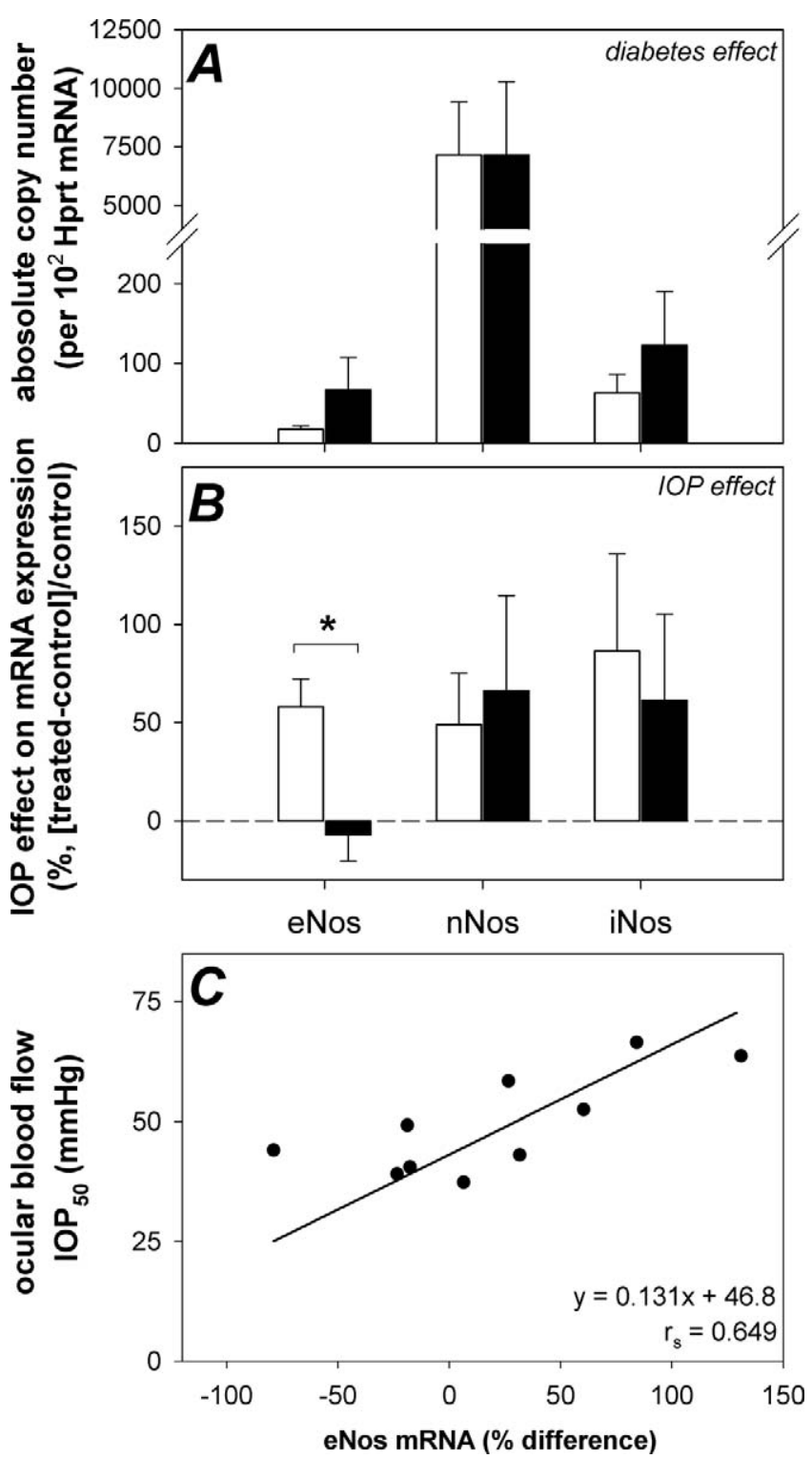

Figure 5. Effect of STZ-diabetes and acute IOP challenge on eNOS, $n N O S$, and $i N O S$ mRNA levels for citrate (unfilled bars) and STZdiabetic eyes (filled bars). (A) Diabetes effect on absolute copy number relative to HPRT (averaged \pm SEM), showing combined control and IOP-treated eyes. (B) Difference in effect of IOP treatment on mRNA expression expressed relative to control eyes (\%, treated-control/ control) for both conditions. Dashed line denotes $0 \%$. Asterisk denotes significance $\left({ }^{*} P<0.05\right)$. (C) Relationship between susceptibility of ocular blood flow to IOP elevation $\left(\mathrm{IOP}_{50}\right)$ and IOP-induced changes in eNos mRNA expression (\%). A Deming linear regression, which takes into account variability in both $y$ - and $x$-axes and is thus more robust for false positives and Spearman correlations (coefficient, $r_{\mathrm{s}}$ ) were employed to describe the relationship (black line) between parameters.

increase in STR susceptibility of $7.4 \mathrm{~mm} \mathrm{Hg}$. This outcome is consistent with a previous study ${ }^{45}$ using the blue field entoptic technique to show that macular blood flow was compromised with smaller IOP elevation in patients with mild or moderate retinopathy (increase of 11 and $8 \mathrm{~mm} \mathrm{Hg}$, respectively) compared with healthy individuals $(15 \mathrm{~mm} \mathrm{Hg})$. This study suggested that the effective autoregulatory range is compromised with increasing severity of diabetic retinopathy. It is worth noting that the LDF measure of blood flow receives contributions from both retinal and choroidal circulations, thus care must be taken when attributing the observed changes to retinal autoregulation.

Impaired autoregulation, as evidenced by diminished retinal vessel reactivity in the eye in response to perturbations, such as oxygen breathing ${ }^{11}$ and flickering light, ${ }^{9,12,13,46}$ have been reported in patients with diabetes. Impaired autoregulation may be associated with vascular changes, such as loss of pericytes and endothelial cells (as reviewed by Fletcher et al. ${ }^{47}$ and Hammes $\left.{ }^{48}\right),{ }^{49}$ which are important in structural support and autoregulation, respectively. Endothelial cells are an important source of both endothelin and nitric oxide; thus, their absence will impair autoregulation.

Several studies suggest that the bioavailability of nitric oxide is altered by hyperglycemia. This change in nitric oxide production has been attributed to disruption of eNOS and nNOS activity. ${ }^{50}$ In this study, acute IOP challenge produced a substantial increase in eNos mRNA expression in citrate control animals but not STZ-rats $(-5.7 \%)$. It appears that an IOP-induced increase in eNos is associated with better blood flow resistance to acute IOP challenge (Fig. 5C). Previous studies have reported that well before the onset of endothelial cell loss, and as early as 1 and 2 weeks after treatment, there is detectable elevation of retinal eNos and nNos levels in STZ-treated rat eyes compared with healthy controls. ${ }^{51,52}$ Although the data were not significant, there was a trend for increased eNos expression in STZdiabetic eyes (Fig. 5A). It has been suggested that hyperglycemia-induced elevation of protein kinase $\mathrm{C}$ activity could lead to the overproduction of nitric oxide, ${ }^{53}$ possibly via upregulation of eNos. It has also been demonstrated that in diabetic mice lacking iNos there was a significant reduction in the number of acellular capillaries and pericyte ghosts. ${ }^{49}$ Thus, iNOS ${ }^{54}$ is also likely to play an important role in the increased sensitivity to IOP challenge observed in our study.

Although our data argue that the functional susceptibility arises from a vascular impairment, we cannot rule out the possibility that in the STZ-diabetic eyes there is (1) reduced neuronal redundancy due to cell loss or (2) hyperglycemiainduced inherent neuronal susceptibility to IOP-related stress. Although retinal cell loss has been described in laboratory and clinical studies, ${ }^{55-57}$ such changes are usually noted 6 weeks after STZ-injection. ${ }^{17,56}$ Kanamori et al. ${ }^{17}$ reported an 8- to 9fold increase in apoptotic cell death after 1 month of STZdiabetes in rats, of which the majority of these TUNEL (terminal transferase dUTP nick end labeling)-positive cells localized to the inner retinal layers. Thus, reduced neuronal redundancy could contribute to our observations. Consistent with this possibility, the pSTR was reduced at baseline in our STZ-diabetic rats, as has been reported elsewhere. ${ }^{41,43}$ Moreover, as IOP increased above $55 \mathrm{~mm} \mathrm{Hg}$, the buffer between the STR amplitude and blood flow was reduced in STZ-diabetic eyes compared with citrate controls (Fig. 4B). This might suggest an additional neuronal susceptibility above the observed impairment in blood flow autoregulation. An inherent neuronal susceptibility might arise from increased oxidative stress as evidenced by the reversal of axonal transport and ion regulation abnormalities in mice overexpressing superoxide dismutase- $2^{58}$ and superoxide dismutase$1,{ }^{59}$ respectively.

\section{Conclusions}

Our study shows that retinal function in STZ-diabetic eyes is more vulnerable to acute IOP challenge. This increased susceptibility occurs very early in the course of STZ-induced 
diabetes, prior to severe retinal dysfunction. This was associated with increased vulnerability of ocular blood flow to IOP elevation, as well as a reduced capacity for diabetic eyes to upregulate eNos in response to IOP stress.

\section{Acknowledgments}

The authors thank Zheng He, Alice Brandli, and Jason Charng for assistance with data collection.

\section{References}

1. Aiello LP. Angiogenic pathways in diabetic retinopathy. $N$ Engl J Med. 2005;353:839-841.

2. Barber AJ. A new view of diabetic retinopathy: a neurodegenerative disease of the eye. Prog Neuropsychopharmacol Biol Psychiatry. 2003;27:283-290.

3. Parisi V, Uccioli L. Visual electrophysiological responses in persons with type 1 diabetes. Diabetes Metab Res Rev. 2001; 17:12-18.

4. Holopigian K, Seiple W, Lorenzo M, Carr R. A comparison of photopic and scotopic electroretinographic changes in early diabetic retinopathy. Invest Ophthalmol Vis Sci. 1992;33: 2773-2780.

5. Bearse MA Jr, Adams AJ, Han Y, et al. A multifocal electroretinogram model predicting the development of diabetic retinopathy. Prog Retin Eye Res. 2006;25:425-448.

6. Harrison WW, Bearse MA Jr, Ng JS, et al. Multifocal electroretinograms predict onset of diabetic retinopathy in adult patients with diabetes. Invest Ophthalmol Vis Sci. 2011; 52:772-777.

7. Bursell SE, Clermont AC, Kinsley BT, Simonson DC, Aiello LM, Wolpert HA. Retinal blood flow changes in patients with insulin-dependent diabetes mellitus and no diabetic retinopathy. Invest Ophthalmol Vis Sci. 1996;37:886-897.

8. Burgansky-Eliash Z, Nelson DA, Bar-Tal OP, Lowenstein A, Grinvald A, Barak A. Reduced retinal blood flow velocity in diabetic retinopathy. Retina. 2010;30:765-773.

9. Lasta M, Pemp B, Schmidl D, et al. Neurovascular dysfunction precedes neural dysfunction in the retina of patients with type 1 diabetes. Invest Ophthalmol Vis Sci. 2013;54:842-847.

10. Cringle SJ, Yu DY, Alder VA, Su EN. Retinal blood flow by hydrogen clearance polarography in the streptozotocininduced diabetic rat. Invest Ophthalmol Vis Sci. 1993;34: 1716-1721.

11. Justesen BL, Mistry P, Chaturvedi N, et al. Retinal arterioles have impaired reactivity to hyperoxia in type 1 diabetes. Acta Ophthalmol. 2010;88:453-457.

12. Nguyen TT, Kawasaki R, Wang JJ, et al. Flicker light-induced retinal vasodilation in diabetes and diabetic retinopathy. Diabetes Care. 2009;32:2075-2080.

13. Mandecka A, Dawczynski J, Vilser W, et al. Abnormal retinal autoregulation is detected by provoked stimulation with flicker light in well-controlled patients with type 1 diabetes without retinopathy. Diabetes Res Clin Pract. 2009;86:51-55.

14. Kern TS, Tang J, Berkowitz BA. Validation of structural and functional lesions of diabetic retinopathy in mice. Mol Vis. 2010;16:2121-2131.

15. Quigley HA, Broman AT. The number of people with glaucoma worldwide in 2010 and 2020. Br J Ophthalmol. 2006;90:262267.

16. Wong VH, Bui BV, Vingrys AJ. Clinical and experimental links between diabetes and glaucoma. Clin Exp Optom. 2011;94:423.

17. Kanamori A, Nakamura M, Mukuno H, Maeda H, Negi A. Diabetes has an additive effect on neural apoptosis in rat retina with chronically elevated intraocular pressure. Curr Eye Res. 2004;28:47-54.

18. Kohzaki K, Vingrys AJ, Armitage JA, Bui BV. Electroretinography in streptozotocin diabetic rats following acute intraocular pressure elevation. Graefes Arch Clin Exp Ophthalmol. 2012; 251:529-535.

19. Arango-Gonzalez B, Schatz A, Bolz S, et al. Effects of combined ketamine/xylazine anesthesia on light induced retinal degeneration in rats. PloS One. 2012;7:e35687.

20. Bui BV, Edmunds B, Cioffi GA, Fortune B. The gradient of retinal functional changes during acute intraocular pressure elevation. Invest Ophthalmol Vis Sci. 2005;46:202-213.

21. Hood DC, Birch DG. The A-wave of the human electroretinogram and rod receptor function. Invest Ophthalmol Vis Sci. 1990;31:2070-2081.

22. Stockton RA, Slaughter MM. B-wave of the electroretinogram. A reflection of ON bipolar cell activity. J Gen Physiol. 1989;93: 101-122.

23. Saszik SM, Robson JG, Frishman LJ. The scotopic threshold response of the dark-adapted electroretinogram of the mouse. J Physiol. 2002;543:899-916.

24. Bui BV, Fortune B. Ganglion cell contributions to the rat fullfield electroretinogram. J Physiol. 2004;555:153-173.

25. Frishman LJ, Sieving PA, Steinberg RH. Contributions to the electroretinogram of currents originating in proximal retina. Vis Neurosci. 1988;1:307-315.

26. Casson RJ, Chidlow G, Wood JP, Osborne NN. The effect of hyperglycemia on experimental retinal ischemia. Arch $O p b$ thalmol. 2004;122:361-366.

27. He Z, Nguyen CT, Armitage JA, Vingrys AJ, Bui BV. Blood pressure modifies retinal susceptibility to intraocular pressure elevation. PloS One. 2012;7:e31104.

28. Chauhan BC, LeVatte TL, Jollimore CA, et al. Model of endothelin-1-induced chronic optic neuropathy in rat. Invest Ophthalmol Vis Sci. 2004;45:144-152.

29. Kiel JW, Shepherd AP. Autoregulation of choroidal blood flow in the rabbit. Invest Ophthalmol Vis Sci. 1992;33:2399-2410.

30. Riva CE, Cranstoun SD, Mann RM, Barnes GE. Local choroidal blood flow in the cat by laser Doppler flowmetry. Invest Ophthalmol Vis Sci. 1994;35:608-618.

31. Lamb TD, Pugh EN Jr. A quantitative account of the activation steps involved in phototransduction in amphibian photoreceptors. J Physiol. 1992;449:719-758.

32. Hood DC, Birch DG. A computational model of the amplitude and implicit time of the b-wave of the human ERG. Vis Neurosci. 1992;8:107-126.

33. Maloney LT. Confidence intervals for the parameters of psychometric functions. Percept Psychophys. 1990;47:127134.

34. Foster DH, Bischof WF. Bootstrap variance estimators for the parameters of small-sample sensory-performance functions. Biol Cybern. 1987;57:341-347.

35. Bui BV, Weisinger HS, Sinclair AJ, Vingrys AJ. Comparison of guinea pig electroretinograms measured with bipolar corneal and unipolar intravitreal electrodes. Doc Ophthalmol. 1998; 95:15-34.

36. Klein R, Klein BE, Moss SE, Cruickshanks KJ. The Wisconsin Epidemiologic Study of Diabetic Retinopathy: XVII. The 14year incidence and progression of diabetic retinopathy and associated risk factors in type 1 diabetes. Ophthalmology. 1998;105:1801-1815.

37. Giove TJ, Deshpande MM, Gagen CS, Eldred WD. Increased neuronal nitric oxide synthase activity in retinal neurons in early diabetic retinopathy. Mol Vis. 2009;15:2249-2258.

38. Goto R, Doi M, Ma N, Semba R, Uji Y. Contribution of nitric oxide-producing cells in normal and diabetic rat retina. Jpn J Ophthalmol. 2005;49:363-370. 
39. Li Q, Verma A, Han PY, et al. Diabetic eNOS-knockout mice develop accelerated retinopathy. Invest Ophthalmol Vis Sci. 2010;51:5240-5246.

40. Abu El-Asrar AM, Meersschaert A, Dralands L, Missotten L, Geboes K. Inducible nitric oxide synthase and vascular endothelial growth factor are colocalized in the retinas of human subjects with diabetes. Eye (Lond). 2004;18:306313.

41. Kohzaki K, Vingrys AJ, Bui BV. Early inner retinal dysfunction in streptozotocin-induced diabetic rats. Invest Ophthalmol Vis Sci. 2008;49:3595-3604.

42. Wong VH, Vingrys AJ, Bui BV. Glial and neuronal dysfunction in streptozotocin-induced diabetic rats. J Ocul Biol Dis Infor. 2011;4:42-50.

43. Ly A, Yee P, Vessey KA, Phipps JA, Jobling AI, Fletcher EL. Early inner retinal astrocyte dysfunction during diabetes and development of hypoxia, retinal stress, and neuronal functional loss. Invest Ophthalmol Vis Sci. 2011;52:93169326.

44. Bui BV, Kalloniatis M, Vingrys AJ. The contribution of glycolytic and oxidative pathways to retinal photoreceptor function. Invest Ophthalmol Vis Sci. 2003;44:2708-2715.

45. Sinclair SH, Grunwald JE, Riva CE, Braunstein SN, Nichols CW, Schwartz SS. Retinal vascular autoregulation in diabetes mellitus. Ophthalmology. 1982;89:748-750.

46. Lecleire-Collet A, Audo I, Aout M, et al. Evaluation of retinal function and flicker light-induced retinal vascular response in normotensive patients with diabetes without retinopathy. Invest Ophthalmol Vis Sci. 2011;52:2861-2867.

47. Fletcher EL, Phipps JA, Wilkinson-Berka JL. Dysfunction of retinal neurons and glia during diabetes. Clin Exp Optom. 2005;88:132-145

48. Hammes HP. Pericytes and the pathogenesis of diabetic retinopathy. Horm Metab Res. 2005;37:39-43.

49. Zheng L, Du Y, Miller C, et al. Critical role of inducible nitric oxide synthase in degeneration of retinal capillaries in mice with streptozotocin-induced diabetes. Diabetologia. 2007;50: 1987-1996.

50. Sobrevia L, Mann GE. Dysfunction of the endothelial nitric oxide signalling pathway in diabetes and hyperglycaemia. Exp Physiol. 1997;82:423-452.

51. Joussen AM, Poulaki V, Qin W, et al. Retinal vascular endothelial growth factor induces intercellular adhesion molecule-1 and endothelial nitric oxide synthase expression and initiates early diabetic retinal leukocyte adhesion in vivo. Am J Pathol. 2002;160:501-509.

52. Takeda M, Mori F, Yoshida A, et al. Constitutive nitric oxide synthase is associated with retinal vascular permeability in early diabetic rats. Diabetologia. 2001;44:1043-1050.

53. Williamson JR, Chang K, Frangos M, et al. Hyperglycemic pseudohypoxia and diabetic complications. Diabetes. 1993; 42:801-813.

54. Berkowitz BA, Luan H, Gupta RR, et al. Regulation of the early subnormal retinal oxygenation response in experimental diabetes by inducible nitric oxide synthase. Diabetes. 2004; 53:173-178.

55. Nakamura M, Kanamori A, Negi A. Diabetes mellitus as a risk factor for glaucomatous optic neuropathy. Ophthalmologica. 2005;219:1-10.

56. Barber AJ, Lieth E, Khin SA, Antonetti DA, Buchanan AG, Gardner TW. Neural apoptosis in the retina during experimental and human diabetes. Early onset and effect of insulin. $J$ Clin Invest. 1998;102:783-791.

57. Bui BV, Loeliger M, Thomas M, et al. Investigating structural and biochemical correlates of ganglion cell dysfunction in streptozotocin-induced diabetic rats. Exp Eye Res. 2009;88: 1076-1083.

58. Sharma R, Buras E, Terashima T, et al. Hyperglycemia induces oxidative stress and impairs axonal transport rates in mice. PloS One. 2010;5:e13463.

59. Berkowitz BA, Gradianu M, Bissig D, Kern TS, Roberts R. Retinal ion regulation in a mouse model of diabetic retinopathy: natural history and the effect of $\mathrm{Cu} / \mathrm{Zn}$ superoxide dismutase overexpression. Invest Ophthalmol Vis Sci. 2009;50:2351-2358.

60. Kaur C, Sivakumar V, Foulds WS. Early response of neurons and glial cells to hypoxia in the retina. Invest Ophthalmol Vis Sci. 2006;47:1126-1141.

61. Kim WT, Suh ES. Retinal protective effects of resveratrol via modulation of nitric oxide synthase on oxygen-induced retinopathy. Korean J Opbthalmol. 2010;24:108-118.

62. Ward MM, Jobling AI, Kalloniatis M, Fletcher EL. Glutamate uptake in retinal glial cells during diabetes. Diabetologia. 2005; 48:351-360. 